\title{
Cinética de secagem de resíduos de jaca (Artocarpus heterophyllus Lam.)
}

\author{
Drying kinetics of jackfruit residues (Artocarpus heterophyllus Lam.) \\ Cinética de secado de residuos la jack (Artocarpus hetrophyllus Lam.)
}

\section{Resumo}

A jaca é consumida na forma in natura ou processada, contribuindo para a geração de resíduos. Tecnologias de processamento, como a secagem, são aplicadas visando produtos de alta qualidade atendendo às exigências do mercado. Assim, objetivou-se estudar a cinética de secagem de resíduos de jaca em estufa à 40,50 e $60{ }^{\circ} \mathrm{C}$ e em forno de micro-ondas, e ajustar modelos matemáticos aos dados experimentais. As cinéticas de secagem foram determinadas pesando-se as amostras em intervalos regulares, até que atingissem o equilíbrio, sendo então determinadas as massas secas. O modelo Wang \& Singh representou à secagem em estufa em todas as temperaturas estudadas, apresentando os melhores valores de coeficiente de determinação e menores desvios quadráticos médios e qui-quadrados. Casca, eixo central, mesocarpo e semente secos à $60{ }^{\circ} \mathrm{C}$ apresentaram teor de água de 5,65, 4,94, 6,42 e 6,36\% após 240, 300, 360, 300 min, respectivamente. O modelo Page representou à secagem em forno de microondas e a rampa de aquecimento das farináceas foram de 3 ciclos de 5 min à potência de $100 \%$. Casca, eixo central, mesocarpo e semente apresentaram teor de água final de 9,57, 7,48, 7,47 e 6,24\% após a secagem por micro-ondas. Portanto, a utilização dos resíduos de jaca torna-se relevante, visto que podem ser processados para a geração de novos produtos alimentícios.

Palavras-chave: Tecnologia de processamento; Modelagem matemática; Reaproveitamento agrícola; Produtos alimentícios.

\begin{abstract}
The jackfruit is consumed in natura or processed form, contributing to the generation of waste. Processing technologies, such as drying, are applied aiming at high quality products meeting market demands. Thus, the aim was to study the drying kinetics of jackfruit residues in an oven at 40,50 and $60^{\circ} \mathrm{C}$ and in a microwave oven, and to adjust mathematical models to experimental data. The drying kinetics were determined by weighing the samples at regular intervals, until they reached equilibrium, and then the dry masses were determined. The Wang \& Singh model represented oven drying at all temperatures studied, presenting the best values of coefficient of determination and the lowest mean square and chi-square deviations. Bark, central axis, mesocarp and seed dried at $60{ }^{\circ} \mathrm{C}$ had a water content of 5.65, 4.94, 6.42 and $6.36 \%$ after 240, 300, 360, $300 \mathrm{~min}$, respectively. The Page model represented the drying in a microwave oven and the heating ramp of the farinaceous was 3 cycles of 5 min at $100 \%$ power. Bark, central axis, mesocarp and seed had final water content of 9.57, 7.48, 7.47 and $6.24 \%$ after microwave drying. Therefore, the use of jackfruit residues becomes relevant, since they can be processed to generate new food products.
\end{abstract}


Keywords: Processing technology; Mathematical modeling; Agricultural reuse; Food products.

\section{Resumen}

La jack se consume en forma natural o procesada, contribuyendo a la generación de residuos. Las tecnologías de procesamiento, como el secado, se aplican con el objetivo de obtener productos de alta calidad que satisfagan las demandas del mercado. Así, el objetivo era estudiar la cinética de secado de los residuos de jack en un horno a 40,50 y $60{ }^{\circ} \mathrm{C}$ y en un horno microondas, y ajustar los modelos matemáticos a los datos experimentales. La cinética de secado se determinó pesando las muestras a intervalos regulares, hasta que alcanzaron el equilibrio, y luego se determinaron las masas secas. El modelo de Wang \& Singh representó el secado en horno a todas las temperaturas estudiadas, presentando los mejores valores de coeficiente de determinación y las desviaciones cuadradas y jicuadradas más bajas. La corteza, el eje central, el mesocarpio y la semilla secados a $60^{\circ} \mathrm{C}$ tuvieron un contenido de agua de 5.65, 4.94, 6.42 y $6.36 \%$ después de 240,300,360, $300 \mathrm{~min}$, respectivamente. El modelo de Page representó el secado en horno microondas y la rampa de calentamiento de la farinácea fue de 3 ciclos de 5 min al 100\% de potencia. La corteza, el eje central, el mesocarpio y la semilla tuvieron un contenido final de agua de 9.57, 7.48, 7.47 y $6.24 \%$ después del secado por microondas. Por tanto, cobra relevancia el uso de residuos de jack, ya que pueden ser procesados para generar nuevos productos alimenticios.

Palabras clave: Tecnología de procesamiento; Modelo matematico; Reutilización agrícola; Productos alimenticios.

\section{Introdução}

A jaqueira (Artocarpus heterophyllus Lam.), pertencente à Família Moraceae, é popularmente cultivada em países tropicais (Baliga, et al., 2011) e, no Brasil, é comum em regiões litorâneas que se estende do sul da Bahia até a Paraíba (Dórea, et al., 2013). Apresenta característica de sazonalidade bem específica, marcada pela concentração da oferta no período de dezembro a abril e sua capacidade de produção é estimada entre 200 e 500 frutas anualmente (Souza, et. al., 2009; Haque, et al., 2015). É, portanto, uma fruteira de grande potencial para a indústria alimentícia e agroindustrial devido a sua capacidade de desenvolvimento e alta produtividade (Swami \& Kalse, 2018).

A jaca é uma fruta que ainda não alcançou o mercado brasileiro, por isso, não há dados oficiais de sua cultura no estado da Paraíba. Dessa forma, sua comercialização informal se dá em feiras livres ou mesmo à beira-de-estradas (Sousa, et al., 2016a), sendo atrativa em virtude de seu aroma e sabor, e também é uma boa fonte de nutrientes, tais como vitaminas, proteínas, sais minerais e fibras quando comparada a outras frutas (Baliga, et al., 2011).

A polpa da jaca é consumida in natura, mas devido à natureza perecível, pode ser processada como geleias, compotas, polpas de frutas, sucos e refrigerantes, e entre os principais resíduos gerados durante o processamento estão a casca, eixo central, mesocarpo e sementes que possuem nutrientes e baixo teor calórico (Swami \& Kalse, 2018). As sementes, por sua vez, são consumidas torradas, cozidas e processadas sob a forma de farinha para a elaboração de diversos produtos de panificação e confeitaria (Hossain, 2014; Madruga, et al., 2014; Silva, et al., 2020c). Os demais componentes da fruta são utilizados como ração animal ou descartados (Silva, et al., 2007; Govindaraj, et al., 2017).

A utilização dos resíduos de jaca visando o aproveitamento na indústria alimentícia e agroindustrial como uma alternativa de elaborar novos produtos a fim de evitar o desperdício e a produção de lixo orgânico, além de agregar valor nutricional, vem sendo investigada (Souza, et al., 2020a; Sousa, et al., 2020a). Uma opção viável é a produção de farinhas alimentícias por meio do processo de secagem, uma vez que esses resíduos processados representam um potencial socioeconômico e alimentício, constituindo-se numa alternativa ao incremento da renda familiar a pequenos e micro indústrias, contribuindo para a sustentabilidade, geração de emprego e renda (Sousa, et al., 2020a; Sousa, et al., 2020b).

Assim, a secagem de produtos alimentícios visa a redução do seu volume e de sua massa durante a remoção de água livre. Esse processo é amplamente utilizado na indústria visando obter um produto de alta qualidade que atenda às exigências do mercado, uma vez que poderá ser utilizado em períodos sem disponibilidade da fruta. Entre as variáveis que mais influenciam esse fenômeno, destaca-se o tempo e a temperatura (Barbosa \& Lobato, 2016; Tadini, et al., 2016). Matematicamente, o fenômeno de secagem é estimado por um sistema de equações diferenciais que representam os balanços 
de massa, energia e quantidade de movimento. Associado a esses modelos, uma série de equações empíricas devem ser empregadas para que esse fenômeno não linear possa ser representado, pois esses estudos são indispensáveis na predição dos fenômenos de transferência de massa e calor (Arruda, et al., 2009; Souza, et al., 2019).

A partir dos dados de cinética de secagem, modelos preditivos de comportamento bem estabelecidos descrevem o processo de diversos produtos e resíduos agrícolas, haja vista a grande diversidade de estrutura e composição dos materiais biológicos e a influência que essas características exercem nos fenômenos de transferência de calor e massa (Mendonça, et al., 2015; Barbosa \& Lobato, 2016). Dentre esses produtos, pode-se citar o estudo da cinética de secagem de: frutos de gueroba (Syagrus oleracea) (Jorge, et al., 2021), mamão cv. Formosa (Carica papaya L.) (Ferreira, et al., 2020), cajá (Spondias mombin L.) (Pinheiro, et al., 2020), pera argentina (Pyrus communis L.) e pera Willians (Pyrus communis 'Williams') (Souza, et al., 2020b); maracujá (Passiflora edulis Sims) (Sousa et al., 2020c); kiwi amarelo (Actinidia chinensis) e kiwi verde (Actinidia deliciosa) (Godoi, et al., 2020); resíduo de acerola (Malpighia emarginata DC) (Sousa, et al., 2020d), sementes germinadas de jaca (Leite, et al., 2019), eixo central e pívide de jaca (Sousa, et al., 2016a).

O método de secagem e o uso de secadores dependem, em geral, dos níveis de produção e da qualidade do produto que se deseje obter, assim, são fundamentais o desenvolvimento e a utilização de novos métodos e processos de secagem (Correia, et al., 2015). Portanto, o objetivo desse trabalho foi estudar a cinética de secagem dos resíduos casca, eixo central, mesocarpo e semente de jaca, por secagem em estufa de circulação de ar à 40,50 e $60{ }^{\circ} \mathrm{C}$ e secagem por forno de micro-ondas, assim como, ajustar modelos matemáticos aos dados experimentais, visando a elaboração de produtos farináceos que possam ser utilizados na formulação de produtos alimentícios.

\section{Metodologia}

O presente trabalho apresenta caráter quali-quantitativo, exploratório e laboratorial (Pereira, et al., 2018) e foi realizado no Laboratório de Armazenamento e Processamento de Produtos Agrícolas, Centro de Tecnologia e Recursos Naturais, e no Laboratório de Bioquímica e Biotecnologia de Alimentos, Centro de Educação e Saúde, ambos da Universidade Federal de Campina Grande. Foram utilizadas jacas da variedade "mole" adquiridas no sítio Bujari, município de Cuité-PB. Escolheu-se as frutas maduras de acordo com a aparência visual da casca verde escurecida e pela consistência macia.

Previamente à secagem, as jacas foram lavadas em água corrente sanitizadas em solução com hipoclorito de sódio (200 ppm) por $15 \mathrm{~min}$, enxaguadas e dispostas em papel toalha a fim de eliminar a água residual. Posteriormente, foram seccionadas manualmente para separação de seus componentes e os resíduos casca, eixo central, mesocarpo e semente foram triturados, individualmente, em liquidificador industrial em velocidade máxima, por um período de aproximadamente 20 min.

As secagens dos resíduos de jaca foram realizadas em estufa de circulação de ar e forno de micro-ondas e, para isso, utilizou-se $40 \mathrm{~g}$ de cada resíduo. Todos os experimentos foram realizados em triplicata. Determinou-se o teor de água dos resíduos em estufa à $105^{\circ} \mathrm{C}$, de acordo com a metodologia do Instituto Adolfo Lutz (IAL, 2008).

A faixa de temperatura estudada, para a secagem em estufa, foi determinada com base na literatura específica a fim de evitar perdas da qualidade física e química dos resíduos de jaca, além de serem empregadas usualmente para produtos alimentícios (Souza, et al., 2009; Oliveira, et al., 2015a; Pinheiro, et al., 2020; Jorge, et al., 2021). Os resíduos foram distribuídos, individualmente, em bandejas circulares de alumínio, com dimensões $24 \mathrm{~cm}$ de diâmetro e $2 \mathrm{~cm}$ de altura e submetidos à 40,50 e $60^{\circ} \mathrm{C}$. As cinéticas de secagem foram determinadas pesando-se as amostras em intervalos regulares de $5,15,30$ e $60 \mathrm{~min}$, até que atingissem o equilíbrio, sendo então determinadas as massas secas.

Para a secagem em forno de micro-ondas, utilizou-se a potência de $100 \%$ (2450 MHz e $700 \mathrm{~W}$ ). No estudo de calibração e mapeamento do mesmo forno utilizado nesse estudo, Oliveira, et al. (2015b) constataram que o ponto máximo de 
incidência de radiação se deu na posição central do forno. As cinéticas de secagem foram determinadas a partir das leituras contínuas em relação à perda de massa de cada resíduo e realizadas em intervalos regulares de 2 em 2 min até que as amostras atingissem peso constante.

Finalizou-se as secagens de cada tratamento quando as massas das amostras não variaram mais de $0,010 \mathrm{~g}$ em três pesagens consecutivas (Oliveira, et al., 2015a), utilizando uma balança digital semi-analítica (Weblabosp, modelo L303i) com 0,001 g de resolução. De acordo com a Equação 1, determinou-se a razão do teor de água das amostras dos resíduos de jaca durante as secagens em estufa, nas diferentes condições de temperatura, e forno de micro-ondas.

$$
R X=\frac{X-X_{e}}{X_{i}-X_{e}}
$$

Em que:

RX - razão do teor de água da amostra (adimensional);

$\mathrm{X}$ - teor de água da amostra em um determinado tempo de secagem (b.s.);

$\mathrm{X}_{\mathrm{i}}$ - teor de água inicial da amostra (b.s.);

$\mathrm{X}_{\mathrm{e}}$ - teor de água de equilíbrio da amostra (b.s.).

Tanto em estufa quanto no forno de micro-ondas, diferentes modelos propostos na literatura foram ajustados na previsão da secagem dos resíduos de jaca (Tabela 1).

Tabela 1. Modelos matemáticos utilizados para predizer o fenômeno de secagem.

\begin{tabular}{|c|c|c|c|}
\hline Designação do modelo & Modelo & Equação & Referências \\
\hline Newton & $\mathrm{RX}=\exp (-\mathrm{k} \cdot \mathrm{t})$ & (2) & (O’Callaghan, 1971) \\
\hline Page & $\mathrm{RX}=\exp \left(-\mathrm{k} \cdot \mathrm{t}^{\mathrm{n}}\right)$ & (3) & (Overhults, et al., 1973) \\
\hline Henderson e Pabis & $\mathrm{RX}=\mathrm{a}$ exo $(-\mathrm{k} . \mathrm{t})$ & (4) & (Henderson \& Pabis, 1962) \\
\hline Logaritmo & $\mathrm{RX}=\mathrm{a} \exp (-\mathrm{k} \cdot \mathrm{t})+\mathrm{c}$ & (5) & (Yagcioglu, et al., 1999) \\
\hline Dois Termos & $\mathrm{RX}=\mathrm{a} \exp \left(-\mathrm{k}_{0} . \mathrm{t}\right)+\mathrm{b} \exp (-\mathrm{k} . \mathrm{t})$ & (6) & (Henderson, 1974) \\
\hline Wang \& Singh & $\mathrm{RX}=1+\mathrm{at}+\mathrm{bt}^{2}$ & (7) & (Wang \& Singh, 1978) \\
\hline
\end{tabular}

onde: rx - razão do teor de água, adimensional; a, b, ck, k0, k1 - parâmetros dos modelos; t -tempo de secagem, min. Fonte: Autores.

Os dados experimentais da secagem dos resíduos de jaca foram submetidos à análise de regressão e seleção do modelo matemático que melhor representou as curvas de secagem. Para o ajuste dos modelos matemáticos, foi realizada análise de regressão não linear pelo método Quasi-Newton, utilizando-se o software STATISTICA 8.0 ${ }^{\circledR}$ (StatSoft, Inc. 2007). A escolha do melhor modelo baseou-se nos seguintes parâmetros estatísticos: coeficiente de determinação ( $\mathrm{R}^{2}$ ) (Equação 8), desvio quadrático médio (DQM) (Equação 9) e qui-quadrado ( $\left.\chi^{2}\right)$ (Equação 10).

$$
\begin{aligned}
& \mathrm{R}^{2}=1-\left(\frac{\sum_{\mathrm{i}=1}^{\mathrm{n}}\left(\mathrm{Rx}_{\text {pred } \mathrm{i}}-\mathrm{RX}_{\text {exp } \mathrm{i}}\right)^{2}}{\sum_{\mathrm{i}=1}^{\mathrm{n}}\left(\mathrm{Rx}_{\exp \mathrm{i}}-\mathrm{RX}_{\text {pred } \mathrm{i}}\right)^{2}}\right) \\
& \mathrm{DQM}=\left[\frac{1}{\mathrm{n}} \sum_{\mathrm{i}=1}^{\mathrm{n}}\left(\mathrm{RX}_{\mathrm{pred}, \mathrm{j}}-\mathrm{RX}_{\mathrm{exp}, \mathrm{i}}\right)^{2}\right]^{\frac{\mathrm{m}}{2}} \\
& \chi^{2}=\frac{1}{n-N} \sum_{i=1}^{n}\left(\mathrm{RX}_{\text {exp }, \mathrm{i}^{\mathrm{i}}}-\mathrm{RX}_{\mathrm{pred}, \mathrm{i}}\right)^{2}
\end{aligned}
$$


Onde:

$\mathrm{R}^{2}$ - coeficiente de determinação;

DQM - desvio quadrático médio;

$\chi^{2}$ - qui-quadrado;

$\mathrm{RX}_{\text {pred,i }}$ - razão do teor de água predito pelo modelo;

$\mathrm{RX}_{\text {exp,i }}$ - razão do teor de água experimental;

n - número de observações;

$\mathrm{N}$ - número de constantes do modelo.

Após a realização das cinéticas de secagem em estufa e forno de micro-ondas, selecionou-se as melhores condições de temperatura e tempo de secagem, bem como, a rampa de aquecimento para a elaboração farinácea dos resíduos de jaca. Por fim, os resíduos secos foram triturados em moinho de facas para obtenção dos produtos. Esses foram acondicionados em embalagens de polietileno de baixa densidade, seladas a vácuo e etiquetados para análises físicas e químicas posteriores.

\section{Resultados e Discussão}

Estimar o comportamento de cada alimento durante a redução do teor de água é relevante para o desenvolvimento e aprimoramento de equipamentos de secagem, e para tal utilizam-se modelos matemáticos que possam representar satisfatoriamente a perda de água durante o período de secagem (Oliveira, et al., 2015a). A Tabela 2 mostra os valores de coeficiente de determinação, desvio quadrático médio e qui-quadrado para modelos matemáticos testados para a secagem em estufa dos resíduos casca, eixo central, mesocarpo e semente de jaca.

Para que um modelo seja adequado na descrição de um fenômeno, quanto menor os valores de desvios quadráticos médios e qui-quadrados melhor será o ajuste do modelo às condições de secagem do produto (Günhan, et al., 2005). Valores maiores de coeficientes de determinação indicam melhor representação do fenômeno estudado pelo modelo, mas não podem ser tomados como critério principal para estimativas não lineares (Oliveira, et al., 2018).

De acordo com a Tabela 2, todos os modelos matemáticos para todas as condições de secagens representaram satisfatoriamente a perda de água dos resíduos de jaca durante a secagem em estufa, uma vez que apresentaram coeficientes de determinação acima de 0,9847 , desvios quadrados médios inferiores a 0,0563 e qui-quadrados menores que 0,0034. Segundo Sozzi \& Ramos (2015), quanto mais próximo de 1 o valor de coeficiente de determinação, mais elucidativo é o modelo e melhor ele se ajustará aos dados experimentais. Entretanto, para modelos não lineares, não é uma boa ferramenta de tomada de decisão, sendo necessária a análise conjunta dos três parâmetros estatísticos.

Tendo em vista esses parâmetros estatísticos, verificou-se, pela Tabela 2, que o modelo apto a descrever a secagem do resíduo casca de jaca, em todas as temperaturas, foi o modelo Wang \& Singh, pois apresentou os maiores valores de coeficientes de determinação $(0,9977$ - 0,9986) e os menores desvios quadráticos médios $(0,0081$ - 0,0226) e qui-quadrados (0,0001 - 0,0005). Porém, deve-se ressaltar que os modelos Page e Logaritmo também apresentaram altos valores de coeficientes de determinação, acima de 0,9942, e menores valores de desvios quadráticos médios e qui-quadrado, abaixo de 0,0362 e 0,0013, respectivamente, indicando sua adequação para representar também a cinética de secagem de casca de jaca. 
Tabela 2. Valores de coeficiente de determinação $\left(\mathrm{R}^{2}\right)$, desvio quadrático médio (DQM) e qui-quadrado $\left(\chi^{2}\right)$ para modelos matemáticos de secagem em estufa dos resíduos casca, eixo central, mesocarpo e semente de jaca (Artocarpus heterophyllus Lam.).

\begin{tabular}{|c|c|c|c|c|c|c|c|}
\hline Modelo & \multicolumn{4}{|c|}{ Casca } & \multicolumn{3}{|c|}{ Eixo central } \\
\hline \multirow{4}{*}{ Newton } & $\mathbf{T}\left({ }^{\circ} \mathbf{C}\right)$ & $\mathbf{R}^{2}$ & DQM & $\chi^{2}$ & $\mathbf{R}^{2}$ & DQM & $\chi^{2}$ \\
\hline & 40 & 0,9931 & 0,0421 & 0,0018 & 0,9937 & 0,0404 & 0,0016 \\
\hline & 50 & 0,9925 & 0,0409 & 0,0017 & 0,9929 & 0,0395 & 0,0016 \\
\hline & 60 & 0,9847 & 0,0584 & 0,0034 & 0,9856 & 0,0563 & 0,0032 \\
\hline \multirow{3}{*}{ Page } & 40 & 0,9972 & 0,0268 & 0,0007 & 0,9960 & 0,0320 & 0,0010 \\
\hline & 50 & 0,9983 & 0,0195 & 0,0004 & 0,9981 & 0,0205 & 0,0004 \\
\hline & 60 & 0,9976 & 0,0234 & 0,0005 & 0,9963 & 0,0287 & 0,0008 \\
\hline \multirow{3}{*}{ Henderson e Pabis } & 40 & 0,9940 & 0,0393 & 0,0015 & 0,9939 & 0,0398 & 0,0016 \\
\hline & 50 & 0,9944 & 0,0354 & 0,0013 & 0,9944 & 0,0350 & 0,0012 \\
\hline & 60 & 0,9892 & 0,0490 & 0,0024 & 0,9885 & 0,0501 & 0,0025 \\
\hline \multirow{3}{*}{ Wang e Singh } & 40 & 0,9986 & 0,0191 & 0,0004 & 0,9970 & 0,0279 & 0,0008 \\
\hline & 50 & 0,9977 & 0,0226 & 0,0005 & 0,9972 & 0,0247 & 0,0006 \\
\hline & 60 & 0,9980 & 0,0081 & 0,0001 & 0,9979 & 0,0214 & 0,0005 \\
\hline \multirow{3}{*}{ Logaritmo } & 40 & 0,9973 & 0,0262 & 0,0007 & 0,9972 & 0,0269 & 0,0007 \\
\hline & 50 & 0,9971 & 0,0254 & 0,0006 & 0,9970 & 0,0258 & 0,0007 \\
\hline & 60 & 0,9942 & 0,0362 & 0,0013 & 0,9934 & 0,0381 & 0,0015 \\
\hline \multirow{3}{*}{ Dois Termos } & 40 & 0,9940 & 0,0393 & 0,0015 & 0,9939 & 0,0398 & 0,0016 \\
\hline & 50 & 0,9944 & 0,0354 & 0,0013 & 0,9944 & 0,0350 & 0,0012 \\
\hline & 60 & 0,9892 & 0,0491 & 0,0024 & 0,9886 & 0,0501 & 0,0025 \\
\hline Modelo & \multicolumn{4}{|c|}{ Mesocarpo } & \multicolumn{3}{|c|}{ Semente } \\
\hline \multirow{4}{*}{ Newton } & $\mathbf{T}\left({ }^{\circ} \mathbf{C}\right)$ & $\mathbf{R}^{2}$ & DQM & $\chi^{2}$ & $\mathbf{R}^{2}$ & DQM & $\chi^{2}$ \\
\hline & 40 & 0,9959 & 0,0306 & 0,0009 & 0,9926 & 0,0422 & 0,0018 \\
\hline & 50 & 0,9951 & 0,0322 & 0,0010 & 0,9934 & 0,0387 & 0,0015 \\
\hline & 60 & 0,9896 & 0,0467 & 0,0022 & 0,9868 & 0,0545 & 0,0030 \\
\hline \multirow{3}{*}{ Page } & 40 & 0,9971 & 0,0261 & 0,0007 & 0,9973 & 0,0253 & 0,0006 \\
\hline & 50 & 0,9986 & 0,0174 & 0,0003 & 0,9993 & 0,0125 & 0,0002 \\
\hline & 60 & 0,9975 & 0,0228 & 0,0005 & 0,9976 & 0,0232 & 0,0005 \\
\hline \multirow{3}{*}{ Henderson e Pabis } & 40 & 0,9960 & 0,0303 & 0,0009 & 0,9940 & 0,0381 & 0,0015 \\
\hline & 50 & 0,9963 & 0,0279 & 0,0008 & 0,9959 & 0,0305 & 0,0009 \\
\hline & 60 & 0,9919 & 0,0413 & 0,0017 & 0,9904 & 0,0466 & 0,0022 \\
\hline \multirow{3}{*}{ Wang \& Singh } & 40 & 0,9969 & 0,0266 & 0,0007 & 0,9995 & 0,0114 & 0,0001 \\
\hline & 50 & 0,9977 & 0,0223 & 0,0005 & 0,9941 & 0,0366 & 0,0013 \\
\hline & 60 & 0,9993 & 0,0122 & 0,0001 & 0,9978 & 0,0225 & 0,0005 \\
\hline \multirow{3}{*}{ Logaritmo } & 40 & 0,9991 & 0,0146 & 0,0002 & 0,9982 & 0,0209 & 0,0004 \\
\hline & 50 & 0,9987 & 0,0169 & 0,0003 & 0,9976 & 0,0235 & 0,0006 \\
\hline & 60 & 0,9962 & 0,0281 & 0,0008 & 0,9942 & 0,0362 & 0,0013 \\
\hline
\end{tabular}




\begin{tabular}{llllllll}
\hline \multirow{3}{*}{ Dois Termos } & 40 & 0,9960 & 0,0303 & 0,0009 & 0,9940 & 0,0381 & 0,0015 \\
& 50 & 0,9963 & 0,0279 & 0,0008 & 0,9953 & 0,0305 & 0,0009 \\
& 60 & 0,9919 & 0,0413 & 0,0017 & 0,9904 & 0,0466 & 0,0022 \\
\hline
\end{tabular}

Fonte: Dados da Pesquisa.

Santos, et al. (2020) determinaram a composição centesimal da casca da pitomba (Talisia esculenta) e realizaram a sua cinética de secagem em estufa de circulação de ar forçado $\left(1,5 \mathrm{~m} \cdot \mathrm{s}^{-1}\right)$, nas temperaturas de 50,60 e $70{ }^{\circ} \mathrm{C}$, como também ajustaram modelos matemáticos aos dados experimentais. O modelo matemático Page foi indicado como o de melhor ajuste ao apresentar elevados valores de coeficientes de determinação maior que 0,99 e os menores valores da função qui-quadrado para todas as temperaturas de secagem aplicadas. Pinheiro, et al. (2020) estudaram a cinética de secagem do epicarpo dos frutos de cajá (S. mombin L.) submetido à secagem em temperaturas de $40,50,60$ e $70{ }^{\circ} \mathrm{C}$ até teores de água final de $0,11,0,10,0,09 \mathrm{e}$ 0,08 (b.s), respectivamente, e constataram que o modelo Midilli apresentou o melhor ajuste aos dados experimentais obtidos.

Ainda de acordo com a Tabela 2, verificou-se que, para todos os modelos e condições de secagem do eixo central de jaca nas temperaturas de 40, 50 e $60{ }^{\circ} \mathrm{C}$, os coeficientes de determinação foram superiores a $98 \%$. Em relação ao desvio quadrático médio, os modelos Page, Wang \& Singh e Logaritmo apresentaram valores menores para todas as temperaturas de secagem. Além desses parâmetros estatísticos, os valores do teste do qui-quadrado apresentaram valores muito baixos para o modelo Wang \& Singh $(0,0005-0,0008)$.

Sousa, et al. (2016a) estudaram a secagem do eixo central de jaca em estufa com circulação e renovação de ar em quatro temperaturas diferentes 50, 6070 e $80^{\circ} \mathrm{C}$ utilizando modelos matemáticos para a descrição da quantidade de água no eixo central de jaca e observaram que o modelo Page foi o que obteve os melhores coeficientes de determinação variando de 0,9982 a 0,9994 e os menores qui-quadrados, apresentando um melhor ajuste dos dados da curva em relação aos outros modelos experimentais. Resultado semelhante também foi observado no presente trabalho.

Verifica-se também que, para todos os modelos e condições de secagem do mesocarpo de jaca, o modelo Wang \& Singh foi o que obteve os melhores coeficientes de determinação variando de 0,9969 a 0,9993 e os menores desvios quadráticos médios e qui-quadrados, apresentando um melhor ajuste dos dados da curva em relação aos outros modelos experimentais. Esse mesmo modelo também foi ajustado de forma eficiente por Oliveira, et al. (2018) na secagem do mesocarpo dos frutos de baru (Dipteryx alata Vogel) nas temperaturas de $40,50,60$ e $70{ }^{\circ} \mathrm{C}$.

Os modelos testados se ajustam bem aos dados experimentais de secagem de semente de jaca, apresentando coeficiente de determinação superior a 0,9868 , desvio quadrático médio inferior a 0,0545 e qui-quadrado abaixo de 0,0030 , os quais podem ser utilizados para predizer as curvas cinéticas de secagem. Os modelos Page e Wang \& Singh apresentaram os maiores coeficientes de determinação e menores desvios quadráticos médios e qui-quadrados (Tabela 2).

Leite, et al. (2019) secaram as sementes germinadas de jaca em secador convectivo a 55 , 65 e $75{ }^{\circ} \mathrm{C}$ com velocidades de secagem de ar de 1,0 e 1,3 m. $\mathrm{s}^{-1} \mathrm{e}$ ajustaram diferentes modelos matemáticos aos dados experimentais. O modelo Dois Termos apresentou os melhores parâmetros de ajuste entre os dez modelos matemáticos testados, resultando no modelo mais adequado para representar o comportamento de secagem.

Portanto, a partir da análise dos parâmetros estatísticos apresentados na Tabela 2, escolheu-se o modelo Wang \& Singh para representar o fenômeno de secagem dos resíduos casca, eixo central, mesocarpo e semente de jaca devido a sua maior simplicidade de aplicação. Sousa, et al. (2016b), Smaniotto, et al. (2017) e Souza, et al. (2019), indicaram o mesmo modelo para representar a curva de secagem do bagaço de malte, dos grãos de girassol (Helianthus annuиs L.) e da polpa de batata-doce biofortificada (Ipomoea batatas L.), respectivamente. 
O método empírico é um método de abordagem com base em dados experimentais e na análise adimensional. Os modelos empíricos de secagem apresentam uma relação direta entre o conteúdo médio do teor de água e o tempo de secagem, omitem os fundamentos do processo de secagem e seus parâmetros não têm significado físico e, consequentemente, não oferecem uma visão apurada dos processos importantes que ocorrem durante o fenômeno, embora descrevam as curvas de secagem para determinadas condições experimentais (Keey, 2013). Entre esses modelos, têm-se o modelo Wang \& Singh, tradicionalmente, utilizados nos estudos de secagem de produtos agrícolas (Martinazzo, et al., 2007).

Assim, a Tabela 3 apresenta os valores dos parâmetros do modelo Wang \& Singh, ajustados aos dados experimentais da cinética de secagem em estufa dos resíduos casca, eixo central, mesocarpo e semente de jaca em diferentes temperaturas. Souza, et al. (2019) constataram que os valores encontrados para o parâmetro A diminuiu com o aumento da remoção de água, a mesma tendência foi verificada para todos os resíduos de jaca quando se variou a temperatura de 40 a $60{ }^{\circ} \mathrm{C}$.

Tabela 3. Parâmetros A e B obtidos no modelo Wang \& Singh para a cinética de secagem em estufa dos resíduos casca, eixo central, mesocarpo e sementes de jaca (Artocarpus heterophyllus Lam.) em temperaturas de 40,50 e $60{ }^{\circ} \mathrm{C}$.

\begin{tabular}{lccc}
\hline Resíduos & \multicolumn{3}{c}{ Parâmetros do Modelo Wang \& Singh } \\
\hline \multirow{4}{*}{ Casca } & T $\left({ }^{\circ} \mathbf{C}\right)$ & A & B \\
\cline { 2 - 4 } & 40 & $-0,0041$ & 0,0000 \\
& 50 & $-0,0068$ & 0,0000 \\
& 60 & $-0,0071$ & 0,0000 \\
\hline \multirow{2}{*}{ Eixo } & 40 & $-0,0041$ & 0,0000 \\
& 50 & $-0,0067$ & 0,0000 \\
& 60 & $-0,0074$ & 0,0000 \\
\hline \multirow{3}{*}{ Mesocarpo } & 40 & $-0,0036$ & 0,0000 \\
& 50 & $-0,0064$ & 0,0000 \\
& 60 & $-0,0071$ & 0,0000 \\
\hline \multirow{3}{*}{ Semente } & 40 & $-0,0040$ & 0,0000 \\
& 50 & $-0,0073$ & 0,0000 \\
& 60 & $-0,0078$ & 0,0000 \\
\hline
\end{tabular}

Fonte: Dados da Pesquisa.

A Figura 1 mostra a razão do teor de água estimados pelo modelo Wang \& Singh para a secagem dos resíduos de jaca à 40,50 e $60^{\circ} \mathrm{C}$. Observou-se que as diferentes temperaturas influenciaram a perda de água e a secagem tornou-se mais rápida em temperaturas mais altas. Desempenho semelhante foi verificado também por Souza, et al. (2019). De acordo com Fiorentin, et al. (2010), o aumento da temperatura acelera o processo de secagem o que, consequentemente, resulta em menor tempo de secagem.

A partir da Figura 1, observou-se que os tempos de secagem nas temperaturas de 40,50 e $60{ }^{\circ} \mathrm{C}$ diminuíram com o aumento da temperatura, apresentando maior taxa de retirada de água. Esses comportamentos das cinéticas de secagem dos resíduos de jaca apresentam características semelhantes a maioria dos produtos agrícolas como sementes de crambe (Crambe abyssinica Hochst) (Costa, et al., 2015), polpa de pequi (Caryocar coriaceum Wittm) (Sousa, et al., 2017) e mesocarpo de baru (Dipteryx alata Vogel) (Oliveira, et al., 2018), existindo uma dependência da velocidade de secagem com a temperatura do ar. 
Figura 1. Valores experimentais da razão do teor de água estimados pelo modelo Wang \& Singh para a secagem, em estufa, dos resíduos casca, eixo central, mesocarpo e semente de jaca (Artocarpus heterophyllus Lam.) em diferentes condições de temperatura.

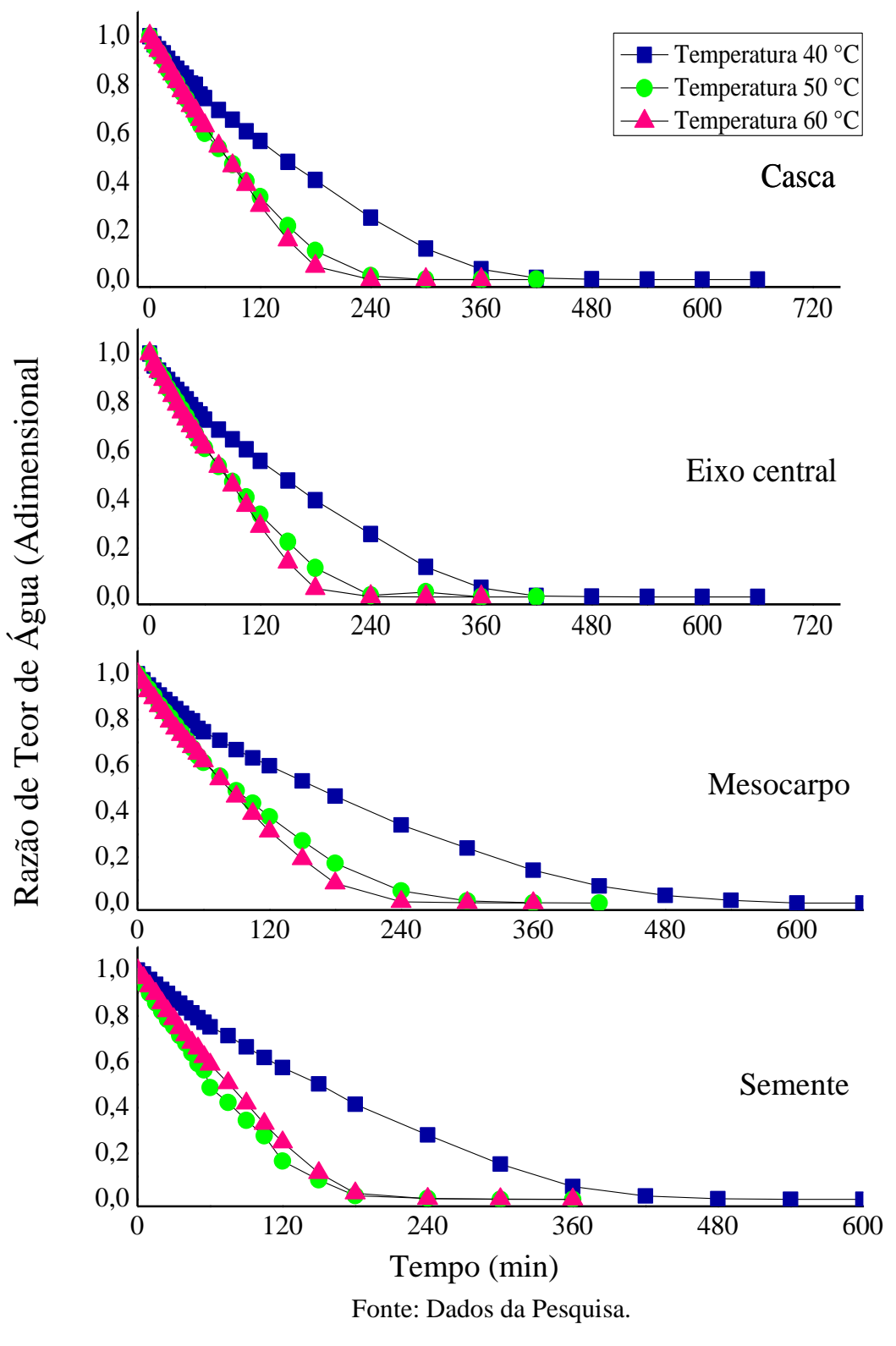

Com o avanço da tecnologia, a secagem por forno de micro-ondas tem assumido cada vez mais importância no processo de secagem do que os convencionais, uma vez que sua aplicação se torna vantajosa devido à taxa de secagem elevada e energia de processamento minimizada com redução do nível de água (Sharma \& Prasad, 2004; Khodja, et al., 2020). Assim, efetuou-se também a secagem dos resíduos de jaca em forno de micro-ondas. A Tabela 4 mostra os valores de coeficiente de determinação, desvio quadrático médio e qui-quadrado para modelos matemáticos testados para a secagem, em forno de microondas, dos resíduos casca, eixo central, mesocarpo e semente de jaca. 
Tabela 4. Valores de coeficiente de determinação $\left(\mathrm{R}^{2}\right)$, desvio quadrático médio (DQM) e qui-quadrado $\left(\chi^{2}\right)$ para modelos matemáticos de secagem em forno de micro-ondas dos resíduos casca, eixo central, mesocarpo e semente de jaca (Artocarpus heterophyllus Lam.).

\begin{tabular}{lcccccc}
\hline \multicolumn{1}{c}{ Modelo } & \multicolumn{3}{c}{ Casca } & \multicolumn{3}{c}{ Eixo central } \\
\cline { 2 - 7 } Newton & $\mathbf{R}^{\mathbf{2}}$ & $\mathbf{D Q M}$ & $\chi^{\mathbf{2}}$ & $\mathbf{R}^{\mathbf{2}}$ & $\mathbf{D Q M}$ & $\chi^{\mathbf{2}}$ \\
\cline { 2 - 7 } Page & 0,9839 & 0,0605 & 0,0037 & 0,9910 & 0,0443 & 0,0020 \\
Henderson e Pabis & 0,9990 & 0,0153 & 0,0002 & 0,9997 & 0,0080 & 0,0001 \\
Wang \& Singh & 0,9869 & 0,0547 & 0,0003 & 0,9924 & 0,0408 & 0,0017 \\
Logaritmo & 0,9988 & 0,0164 & 0,0003 & 0,9959 & 0,0300 & 0,0009 \\
Dois Termos & 0,9944 & 0,0358 & 0,0013 & 0,9960 & 0,0296 & 0,0009 \\
\hline \multicolumn{1}{c}{ Modelo } & 0,9869 & 0,0547 & 0,0030 & 0,9924 & 0,0408 & 0,0017 \\
\hline \multicolumn{3}{c}{ Mesocarpo } & & & Semente & \\
Newton & & $\mathbf{D Q M}$ & $\chi^{2}$ & $\mathbf{R}^{2}$ & $\mathbf{D Q M}$ & $\chi^{2}$ \\
Page & 0,9886 & 0,0482 & 0,0023 & 0,9928 & 0,0379 & 0,0014 \\
Henderson e Pabis & 0,9995 & 0,0105 & 0,0001 & 0,9998 & 0,0062 & 0,00004 \\
Wang \& Singh & 0,9903 & 0,0446 & 0,0020 & 0,9939 & 0,0348 & 0,0012 \\
Logaritmo & 0,9780 & 0,0669 & 0,0045 & 0,9837 & 0,0569 & 0,0032 \\
Dois Termos & 0,9923 & 0,0397 & 0,0016 & 0,9958 & 0,0298 & 0,0008 \\
\hline
\end{tabular}

Fonte: Dados da Pesquisa.

A partir da Tabela 4, observou-se que todos os modelos testados se ajustaram aos dados experimentais de secagem dos resíduos de jaca, apresentando coeficiente de determinação superior a 0,9780, desvios quadráticos médios inferiores a 0,06069 e qui-quadrados menores que 0,0045, podendo ser utilizados para predizer as curvas de cinética de secagem dos resíduos casca, eixo central, mesocarpo e semente de jaca em forno de micro-ondas. Dentre os modelos testados, o modelo Page foi selecionado por apresentar uma equação mais simples, conforme relato por Costa, et al. (2015), estimando-se as curvas de cinética de secagem dos resíduos de jaca. Verificou-se também que apresentou o maior coeficiente de determinação e menores desvio quadrático médio e qui-quadrado.

Sousa, et al. (2020d) descreveram a cinética de secagem do resíduo de acerola em forno de micro-ondas e verificaram que os diferentes modelos matemáticos se ajustaram bem aos dados experimentais, no entanto, o modelo Midilli apresentou maior coeficiente de determinação e menor desvio quadrático médio, indicando a representação satisfatória do fenômeno de secagem do resíduo de acerola.

Na Tabela 5, são apresentados os valores dos parâmetros do modelo Page ajustados aos dados observados durante a secagem dos resíduos de jaca em forno de micro-ondas. O parâmetro de secagem $\mathrm{K}$ do modelo representa as condições externas de secagem e pode ser utilizada como uma aproximação para caracterizar o efeito da temperatura, enquanto que o parâmetro N reflete a resistência interna do produto à secagem (Babalis \& Belessiotis, 2004; Araujo, et al., 2017). 
Tabela 5. Parâmetros do modelo Page para a cinética de secagem em forno de micro-ondas dos resíduos casca, eixo central, mesocarpo e sementes de jaca (Artocarpus heterophyllus Lam.).

\begin{tabular}{lcc}
\hline \multicolumn{1}{c}{ Resíduo } & \multicolumn{2}{c}{ Parâmetros do Modelo de Page } \\
\hline \multirow{2}{*}{ Casca } & $\mathbf{K}$ & $\mathbf{N}$ \\
\cline { 2 - 3 } Eixo central & 0,0586 & 1,4797 \\
Mesocarpo & 0,1028 & 1,3699 \\
Semente & 0,0921 & 1,4868 \\
\hline & 0,1096 & 1,3446 \\
\hline
\end{tabular}

Fonte: Dados da Pesquisa.

As curvas de secagem dos resíduos de jaca obtidas em forno de micro-ondas e estimadas pelo modelo Page são apresentadas na Figura 2. A cinética de secagem mostrou que o teor de água diminuiu gradativamente até o final da secagem por micro-ondas.

Figura 2. Valores observados da razão do teor de água estimados pelo modelo Page para a secagem, em forno micro-ondas, dos resíduos casca, eixo central, mesocarpo e semente de jaca (Artocarpus heterophyllus Lam.).

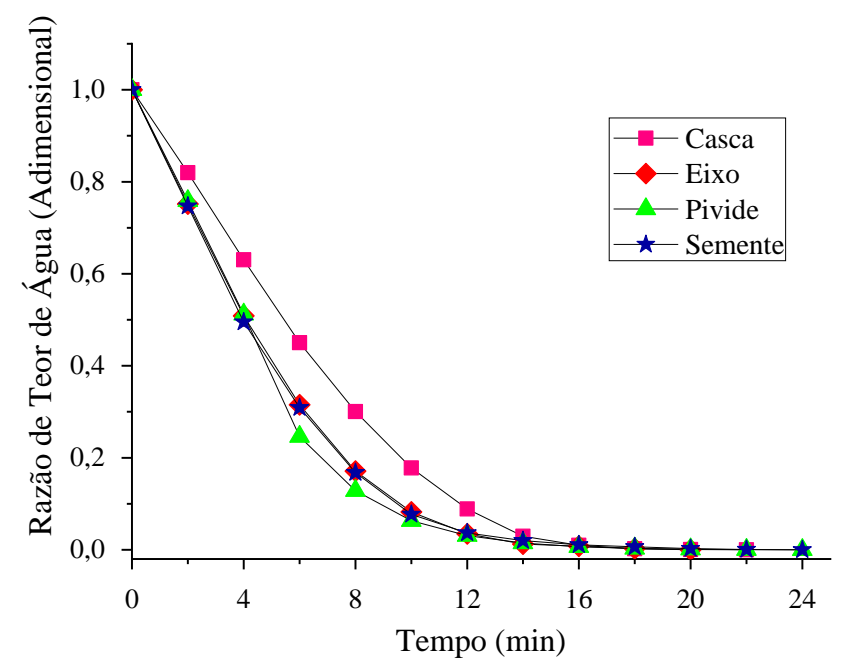

Fonte: Dados da Pesquisa.

Consoante aos valores encontrados na Tabela 5, observou-se que o resíduo casca apresentou valor do parâmetro K do modelo Page inferior aos valores dos demais resíduos de jaca. Já os resíduos eixo central, mesocarpo e semente apresentaram valores muito próximos, por isso a tendência de sobreposição das curvas de secagem desses resíduos, uma vez que, mostra a conformidade entre os dados experimentais e as curvas previstas (Figura 2). Constatou-se ainda que para uma mesma potência de micro-ondas aplicada, os resíduos de jaca apresentaram cinética de secagem semelhante. Resultado similar foi observado por Gonçalves, et al. (2016) na secagem por micro-ondas de três cultivares de mandioca (Manihot esculenta Crantz).

Logo, as secagens dos resíduos de jaca foram eficientes para a redução de água pelos diferentes métodos de secagem utilizados. Entretanto, vale ressaltar que os resultados obtidos a partir dos dois métodos de secagem evidenciaram que, em comparação com o método em estufa, o tempo de secagem necessário para atingir o teor de água de equilíbrio dos resíduos de 
jaca pode ser significativamente reduzido por micro-ondas, pois, de acordo com Khodja, et al. (2020), o princípio usado para a secagem por estufa diferencia-se da secagem por micro-ondas.

Isso se deve ao fato de que, durante a secagem em estufa, o calor é transferido para a superfície do material por condução, convecção e/ou radiação, e conduzido para o interior por condução térmica. Para a secagem por forno de microondas, as micro-ondas penetram no material e interagem com a água, que é uma molécula polar. Como as moléculas de água são dipolares por natureza, elas giram seguindo o campo elétrico que se alterna em frequência muito alta. Essa rotação, produz atrito de moléculas e gera calor dentro do material. Visto que os alimentos são ricos em água, as micro-ondas podem gerar um calor volumétrico significativo. A água aquecida aumenta a taxa de difusão e o gradiente de pressão que facilita a expulsão da água de dentro para fora do material (Khodja, et al., 2020). Dessa forma, a Tabela 6 mostra os valores do teor de água dos resíduos de jaca no tempo inicial e final dos diferentes processos de secagem.

Tabela 6. Valores médios do teor de água dos resíduos casca, eixo central, mesocarpo e semente de jaca (Artocarpus heterophyllus Lam. no tempo inicial e final das secagens em estufa e forno de micro-ondas.

\begin{tabular}{cccccc}
\hline & Teor de água & \multicolumn{5}{c}{ Teor de água final (\%) (b.s) } \\
\cline { 3 - 6 } $\begin{array}{c}\text { inicial } \\
(\boldsymbol{\%})(b . s)\end{array}$ & $\mathbf{4 0}^{\circ} \mathbf{C}$ & $\begin{array}{c}\text { Estufa } \\
\mathbf{5 0}{ }^{\circ} \mathbf{C}\end{array}$ & $\mathbf{6 0}^{\circ} \mathbf{C}$ & Forno de micro-ondas \\
\hline Casca & $81,78 \pm 0,60$ & $12,49 \pm 0,81$ & $8,11 \pm 0,81$ & $5,65 \pm 0,45$ & $9,57 \pm 0,31$ \\
Eixo central & $83,72 \pm 0,74$ & $11,10 \pm 0,37$ & $6,57 \pm 0,84$ & $4,94 \pm 0,22$ & $7,48 \pm 0,45$ \\
Mesocarpo & $82,83 \pm 0,73$ & $17,38 \pm 0,62$ & $6,52 \pm 0,35$ & $6,42 \pm 0,76$ & $7,47 \pm 0,67$ \\
Semente & $74,10 \pm 0,44$ & $10,25 \pm 0,58$ & $5,57 \pm 0,95$ & $6,36 \pm 0,06$ & $6,24 \pm 0,45$ \\
\hline
\end{tabular}

Fonte: Dados da Pesquisa.

Para a obtenção dos produtos farináceos de casca, eixo central, mesocarpo e semente de jaca pelo método estufa, escolheu-se a temperatura de $60{ }^{\circ} \mathrm{C}$, uma vez que, os menores valores de teor de água (Tabela 6) foram alcançados após 240, 300, 360, 300 min, respectivamente. Logo, a utilização da temperatura elevada permite uma secagem mais rápida, fato também observado por Silva, et al. (2020a), Silva, et al. (2020b), Sousa, et al. (2020b) e Sousa et al. (2020c) no processo de secagem de maxixe (Cucumis anguria L.); resíduos agroindustriais provenientes do processamento de polpa de frutas (goiaba (Psidium guajava L.), manga (Mangifera indica L.), acerola (M. emarginata DC), graviola (Annona muricata Linnaeus.), maracujá ( $P$. edulis Sims), caju (Anacardium occidentale L.), cajá (S. mombin L.) e uva (Vitis vinifera L.)); eixo central e pívide de jaca; e casca de jaca, respectivamente.

Para a secagem em forno de micro-ondas, os valores de teor de água final dos resíduos de jaca, como mostrados na Tabela 6, foram alcançados após $16 \mathrm{~min}$. Com base nos resultados da cinética de secagem em forno de micro-ondas, a elaboração dos produtos farináceos seguiu uma rampa de aquecimento por 3 ciclos de $5 \mathrm{~min}$, na potência de $100 \%$ (2450 MHz e $700 \mathrm{~W}$ ) e massa inicial de amostra de 40 g. Após esse tempo, ainda se conseguiu redução no teor de água, no entanto, ocorreu o escurecimento visual das amostras.

Portanto, a utilização ou o aproveitamento integral dos resíduos de jaca torna-se relevante, representando uma importante solução ambiental, visto que esses resíduos podem ser destinados ao processamento como matéria-prima para a geração de novos produtos. Como esses resíduos são materiais de baixo custo, apresentam vantagens econômicas para a obtenção de produtos que poderão, posteriormente, ser testados em formulações de novos produtos de panificação. 


\section{Considerações Finais}

Na secagem em estufa dos resíduos de jaca, houve influência das diferentes temperaturas sobre as curvas de secagem, evidenciando a dependência da velocidade da secagem com a temperatura do ar, uma vez que, o aumento da temperatura ocasionou uma maior taxa de remoção de água nos resíduos. O modelo Wang \& Singh foi o mais indicado para representar o fenômeno de secagem em estufa nas diferentes condições de temperaturas estudadas. Os resíduos casca, eixo central, mesocarpo e semente de jaca secos à $60{ }^{\circ} \mathrm{C}$ apresentaram teor de água de 5,65, 4,94, 6,42 e 6,36\%, respectivamente, após 240 , 300, 360, 300 min, respectivamente. Nessa condição, elaborou-se os produtos farináceos dos resíduos.

No processo de secagem em forno de micro-ondas, o modelo Page foi o mais indicado para representar o fenômeno de secagem dos resíduos. Na mesma potência de micro-ondas utilizada, os resíduos de jaca apresentaram cinéticas de secagem semelhantes. A rampa de aquecimento utilizada para o processo de obtenção das farinhas foi constituída de 3 ciclos de 5 min, potência de $100 \%$ (2450 MHz e $700 \mathrm{~W}$ ) e $40 \mathrm{~g}$ de amostra. Os produtos secos de casca, eixo central, mesocarpo e semente de jaca apresentaram teor de água de 9,57, 7,48, 7,47 e 6,24\% após $16 \mathrm{~min}$.

As secagens dos resíduos casca, eixo central, mesocarpo e semente de jaca foram eficientes para a redução de água pelos métodos de estufa de circulação de ar e forno de micro-ondas. Os modelos matemáticos aplicados auxiliaram na previsão do tempo, melhoria das condições e avaliação da qualidade do processo de secagem. Com isso, a utilização dos resíduos submetidos às tecnologias de processamento se torna interessante, uma vez que, favorece o aproveitamento integral da fruta e a redução dos desperdícios.

Portanto, trabalhos direcionados à elaboração de produtos farináceos a partir dos resíduos casca, eixo central, mesocarpo e semente de jaca utilizando diferentes métodos de secagens já vêm sendo desenvolvidos, e estudos futuros podem ser realizados para avaliar a possibilidade do uso dessas farinhas na elaboração e/ou preparo de diversos produtos alimentícios, sendo necessária além da caracterização física, química e nutricional, a análise sensorial dessas possíveis formulações.

\section{Agradecimentos}

Os autores agradecem à FAPESQ/PRONEX (Concessão 005/2019) do projeto "Processos biotecnológicos para beneficiamento de resíduos agroindustriais" no apoio dado a pesquisa.

\section{Referências}

Araujo, W. D., Goneli, A. L. D., Corrêa, P. C., Hartmann Filho, C. P., \& Martins, E. A. S. (2017). Mathematical modelling of thin-layer drying in peanut fruit. Revista Ciência Agronômica, 48(3), 448-457. 10.5935/1806-6690.20170052

Arruda, E. B., Lobato, F. S., Assis, A. J., \& Barrozo, M. A. S. (2009). Modeling of fertilizer drying in roto-aerated and conventional rotary dryers. Drying Technology, 27(11), 1192-1198. 10.1080/07373930903263129

Babalis, S. J., \& Belessiotis, V. G. (2004). Influence of the drying conditions on the drying constants and moisture diffusivity during the thin-layer drying of figs. Journal of food Engineering, 65(3), 449-458. 10.1016/j.jfoodeng.2004.02.005

Baliga, M. S., Shivashankara, A. R., Haniadka, R., Dsouza, J., \& Bhat, H. P. (2011). Phytochemistry, nutritional and pharmacological properties of Artocarpus heterophyllus Lam (jackfruit): A review. Food Research International, 44(7), 1800-1811. 10.1016/j.foodres.2011.02.035

Barbosa, T. A., \& Lobato, F. S. (2016). Determinação da cinética de secagem de produtos alimentícios usando algoritmos genéticos. Journal of Neotropical Agriculture, 3(3), 28-37. 10.32404/rean.v3i3.1205

Correia, A. F. K., Loro, A. C., Zanatta, S., Spoto, M. H. F., \& Vieira, T. M. F. S. (2015). Effect of temperature, time, and material thickness on the dehydration process of tomato. International Journal of Food Science, 2015. 10.1155/2015/970724

Costa, L. M., Resende, O., Goncalves, D. N., \& de Oliveira, D. E. C. (2015). Mathematical modeling of crambe fruits in thin layer drying. Bioscience Journal, 31(2), 392-403. 10.14393/BJ-v31n2a2015-22340

Dórea, J. R. R., Pereira, L. G. R., Ferreira, A. L., da Silva, T. C., Azevêdo, J. A. G., de Gouvêa, V. N., \& Franco, A. L. C. (2013). Chemical composition and fermentation dynamics of jackfruit silage. Semina: Ciências Agrárias, 34(4), 1967-1976. 10.5433/1679-0359.2013v34n4p1967 
Ferreira, J. P. D. L., Castro, D. S. D., Moreira, I. D. S., Silva, W. P. D., de Figueirêdo, R. M., \& Queiroz, A. J. D. M. (2020). Convective drying kinetics of osmotically pretreated papaya cubes. Revista Brasileira de Engenharia Agrícola e Ambiental, 24(3), 200-208. 10.1590/1807-1929/agriambi.v24n3p200-208

Fiorentin, L. D., Menon, B. T., Alves, J. A., de Barros, S. T. D., Pereira, N. C., \& Módenes, A. N. (2010). Determinação da cinética e das isotermas de secagem do bagaço da laranja. Acta Scientiarum. Technology, 32(2), 147-152. 10.4025/actascitechnol.v32i2.8242

Godoi, A. S., dos Santos, B. M. M., Holsbach, F. M. S., da Silva, W. C., de Lima, T., Werle, L. O., \& Galante, R. M. (2020). Determination of the drying kinetics of yellow kiwi (Actinidia chinensis) and green kiwi (Actinidia delicious). Brazilian Journal of Development, 6(7), 51941-51950. 10.34117/bjdv6n7733

Gonçalves, L. T., Pereira, N. R., Almeida, S. B., Freitas, S. D. J., \& Waldman, W. R. (2017). Microwave-hot air drying applied to selected cassava cultivars: drying kinetics and sensory acceptance. International Journal of Food Science \& Technology, 52(2), 389-397. 10.1111/ijfs.13293

Govindaraj, D., Rajan, M., Hatamleh, A. A., \& Munusamy, M. A. (2018) From waste to high-value product: Jackfruit peel derived pectin/apatite bionanocomposites for bone healing applications. International journal of biological macromolecules, 106, 293-301. 10.1016/j.ijbiomac.2017.08.017

Günhan, T., Demir, V., Hancioglu, E., \& Hepbasli, A. (2005). Mathematical modelling of drying of bay leaves. Energy Conversion and Management, 46, 1667-1679. 10.1016/j.enconman.2004.10.001

Haque, M. A., Begum, R., Shibly, A. Z., Sultana, M. M., \& Khatun, A. (2015). Influence of jackfruit pulp on the quality and shelf life of jackfruit cake. Journal Environmental Science and Natural Resources, 8(1), 59-64. 10.3329/jesnr.v8i1.24672

Henderson, S. M. (1974). Progress in developing the thin-layer drying equation. Transactions of the American Society of Agricultural. 17(6), 1167-1168. $10.13031 / 2013.37052$

Henderson, S. M., \& Pabis, S. (1962). Grain drying theory I: temperature effect on drying coefficient. Journal of Agricultural Research Engineering, 12, 732736.

Hossain, M. T. (2014). Development and quality evaluation of bread supplemented with jackfruit seed flour. International Journal of Nutrition and Food Sciences, 3(5), 484. 10.11648/j.ijnfs.20140305.28

Instituto Adolfo Lutz (São Paulo). (2008). Métodos físico-químicos para análise de alimentos. 4.ed. São Paulo: Instituto Adolfo Lutz, 1020p.

Jorge, A. P. P., Ferreira Junior, W. N., Silva, L. C. M., Oliveira, D. E. C., \& Resende, O. (2021). Drying kinetics of 'gueroba' (Syagrus oleracea) fruit pulp. Brazilian Journal of Agricultural and Environmental Engineering, 25(1), 23-29. 10.1590/1807-1929/agriambi.v25n1p23-29

Khodja, Y. K., Dahmoune, F., Bachir bey, M., Madani, K., \& Khetta, B. (2020). Conventional method and microwave drying kinetics of Laurus nobilis leaves: effects on phenolic compounds and antioxidant activity. Brazilian Journal of Food Technology, 23, e2019214. 10.1590/1981-6723.21419

Keey, R. B. (2013). Drying: Principles and practice. Elsevier.

Leite, D. D. D. F., Queiroz, A. J. D. M., Figueirêdo, R. M. F. D., \& Lima, L. S. L. (2019). Mathematical drying kinetics modeling of jackfruit seeds (Artocarpus heterophyllus Lam.). Revista Ciência Agronômica, 50(3), 361-369. 10.5935/1806-6690.20190043

Madruga, M., Samara, M. A. F., Rafaela, A. S. I., Silva, A. D., Magnani, M., \& Queiroga Neto, V. (2014). Chemical, morphological and functional properties of Brazilian jackfruit (Artocarpus heterophyllus Lam) seeds starch. Food Chemistry, 143, 440-445. doi:10.1016/j.foodchem.2013.08.003

Martinazzo, A. P., Corrêa, P. C., Resende, O., \& Melo, E. C. (2007). Analysis and mathematical description of drying kinetic of lemon grass leaves. Revista Brasileira de Engenharia Agrícola e Ambiental, 11(3), 301-306. 10.1590/S1415-43662007000300009

Mendonça, A. P., Sampaio, P. D. T., Almeida, F. D. A., Ferreira, R. F., \& Novais, J. M. (2015). Determination of drying curves of crabwood in solar dryer. Revista Brasileira de Engenharia Agrícola e Ambiental, 19(4), 382-387. 10.1590/1807-1929/agriambi.v19n4p382-387

O’Callaghan, J. R., Menzies, D. J., \& Bailey, P. H. (1971). Digital simulation of agricultural dryer performance. Journal of Agricultural Engineering Research, 16(3), 223-244. 10.1016/S0021-8634(71)80016-1

Oliveira, G. H. H. D., Aragão, D. M. S., Oliveira, A. P. L. R. D., Silva, M. G., \& Gusmão, A. C. A. (2015a). Modelling and thermodynamic properties of the drying of strawberries. Brazilian Journal of Food Technology, 18(4), 314-321. 10.1590/1981-6723.5315

Oliveira, J. A. M., Macedo, A. D. B., Raulino, J. L. C., Raulino, A. D. M. D., Santana, R. A. C., \& Campos, A. R. N. (2015b). Determinação do teor de água de cactáceas pelos métodos padrão em estufa e micro-ondas. Blucher Chemistry Proceedings, 3(1), 1027-1037.

Oliveira, P. M., Oliveira, D. E. C., Resende, O., \& Silva, D. V. (2018). Study of the drying of mesocarp of baru (Dipteryx alata Vogel) fruits. Revista Brasileira de Engenharia Agrícola e Ambiental, 22(12), 872-877. 10.1590/1807-1929/agriambi.v22n12p872-877

Overhults, D. G., White, G. M., Hamilton, H. E., \& Ross, I. J. (1973). Drying soybeans with heated air. Transactions of the of the American Society of Agricultural, 16(1), 112. 10.13031/2013.37459

Pereira, N. R., Marsaioli Júnior, A., \& Ahrné, L. M. (2007). Effect of microwave power, air velocity and temperature on the final drying of osmotically dehydrated bananas. Journal of Food Engineering, 81(1), 79-87. 10.1016/j.jfoodeng.2006.09.025

Pinheiro, G. K. I., Oliveira, D. E. C., Ferreira Junior, W. N., \& Resende, O. (2020). Drying kinetics of yellow mombin (Spondias mombin L.) epicarp. Revista Brasileira de Engenharia Agrícola e Ambiental, 24(2), 121-127. 10.1590/1807-1929/agriambi.v24n2p121-127 
Santos, N. C., Almeida, R. L. J., Pereira, T. S., Queiroga, A. P. R., Silva, V. M. de A., Amaral, D. S., Almeida, R. D., Ribeiro, V. H. A., Barros, E. R., \& Silva, L. R. I. (2020). Mathematical modeling applied to the drying kinetics of pitomba bark (Talisia esculenta). Research, Society and Development, 9(2), e46921986. 10.33448/rsd-v9i2.1986

Sharma, G., \& Prasad, S. (2004). Effective moisture diffusivity of garlic cloves undergoing microwave-convective drying. Journal of Food Engineering, 65(4), 609-617. 10.1016/j.jfoodeng.2004.02.027

Silva, J. H. V., Jordão Filho, J., Ribeiro, M. L. G., \& Silva, E. L. (2007). Efeitos da inclusão do farelo de sementes de jaqueira (Artocarpus heterophyllus Lam.) na ração sobre a produção, pigmentação da gema e umidade fecal em codornas. Ciência e Agrotecnologia, 31(2), 523-530. 10.1590/S141370542007000200037

Silva, A. P. F., Sousa, A. P. M., Macedo, A. D. B., Dantas, D. L., Oliveira, J. A. M., Almeida, A. F., Santana, R. A. C., \& Campos, A. R. N. (2020a). Obtaining the fuit of the flour maxixe (Cucumis anguria L.) by different drying methods. Brazilian Journal of Development, 6(7), 50983-51000. $10.34117 /$ bjdv6n7-661

Silva, A. P. F., Sousa, A. P. M., Macedo, A. D. B., Dantas, D. L., Costa, J. D., Almeida, A. F., Santana, R. A. C., \& Campos, A. R. N. (2020b). Obtaining floury product from agro-industrial waste by different drying methods. Research, Society and Development, 9(9), e405997334. 10.33448/rsd-v9i9.7334

Silva, J. C. C., Matias, R. S. de L., Oliveira, M. J. S., Araújo, J. de M., \& Viera, V. B. (2020c). Elaboration and sensory evaluation of added cookie from jackfruit seed and vegan dulce de leche. Research, Society and Development, 9(8), e585985757. 10.33448/rsd-v9i8.5757

Smaniotto, T. A. S., Resende, O., Sousa, K. A., Oliveira, D. E. C., \& Campos, R. C. (2017). Drying kinetics of sunflower grains. Revista Brasileira de Engenharia Agrícola e Ambiental, 21(3), 203-208. 10.1590/1807-1929/agriambi.v21n3p203-208

Sousa, H. C., Silva, N. J. P., Pereira, E. M., Silva Filho, C. R. M., \& Souza, W. F. C. (2016a). Use of numerical and analytical solutions in the description of the drying of the central axis of jackfruit. Revista Verde de Agroecologia e Desenvolvimento Sustentável, 11(3), 131-134. 10.18378/rvads.v11i3.4314

Sousa, F. T., Silva, M. A. P., Oliveira, D. E. C., Plácido, G. R., Gagnin, C., Moura, L. C., Souza, D. G., Caliari, M., \& Lima, M.S. (2016b). Modelagem matemática da secagem e propriedades físicas e adaptações do bagaço de malte. Global Science and Technology, 9, 51-61.

Sousa, E. P., Figueirêdo, R. M. F., Gomes, J. P., Queiroz, A. J. M., Castro, D. S., \& Lemos, D. M. (2017). Mathematical modeling of pequi pulp drying and effective diffusivity determination. Revista Brasileira de Engenharia Agrícola e Ambiental, 21, (7), 493-498. 10.1590/1807-1929/agriambi.v21n7p493-498

Sousa, A. P. M., Costa, J. D., Macedo, A. D. B., Dantas, D. L., Oliveira, J. A. M., Almeida, A. F., Santana, R. A. C., \& Campos, A. R. N. (2020a). Physical and chemical characterization of farinaceous product the central axis and pivot of jackfruit. Research, Society and Development, 9(9), e350997333e350997333. 10.33448/rsd-v9i9.7333

Sousa, A. P. M., Campos, A. R. N., Macedo, A. D. B., Dantas, D. L., Apolinário, M. O., \& Santana, R. A. C. (2020b). Quality assessment flour of jacafruit shell. Brazilian Journal of Animal and Environmental Research, 3(3), 1786-1796. 10.34188/bjaerv3n3-094

Sousa, C. F., Sousa, S., Figueiredo, J. S. B., Moraes, M. R. L., Sena Carvalho, I. P. P., Chaves, F. J. F., Mata, M. E. R. M., \& Almeida, G. N. (2020c). Kinetic drying in low food layout of passion fruit, using different additives. Brazilian Journal of Development, 6(9), 70821-70829. 10.34117/bjdv6n9-506

Sousa, A. P. M., Campos, A. R. N., Macedo, A. D. B., Dantas, D. L., Silva, A. P. F., Costa, J. D., \& Santana, R. A. C. (2020d). Mathematical modeling of acerola residence in microwave. Brazilian Journal of Animal and Environmental Research, 3(3), 1797-1806. 10.34188/bjaerv3n3-095

Souza, T. S. A., Chaves, M. A., Bonomo, R. C. F., Soares, R. D., Pinto, E. G., \& Cota, I. R. (2009). Desidratação osmótica de frutículos de jaca (Artocarpus integrifólia L.): aplicação de modelos matemáticos. Acta Scientiarum. Technology, 31(2), 225-230. 10.4025/actascitechnol.v31i2.1026

Souza, D. G., Resende, O., Moura, L. C., Ferreira Junior, W. N., \& Andrade, J. W. S. (2019). Drying kinetics of the sliced pulp of biofortified sweet potato (Ipomoea batatas L.). Engenharia Agrícola, 39(2), 176-181. 10.1590/1809-4430-Eng.Agric.v39n2p176-181/2019

Souza, H. M. S., Silva, E. M., Souza, T. R. L., Mendes, M. L. M., \& Messias, C. M. B O. (2020a). Potential of pulp and jackfruit residues (Artocarpus heterophyllus Lam.) in the preparation of a sustainable sweet. Brazilian Journal of Development, 6(11), 87251-87269. 10.34117/bjdv6n11-229

Souza, P. D., Durante, T. P. Y., Galante, R. M., \& Werle, L. O. (2020b). Drying kinetics of Argentine pear (Pyrus communis L.) and Willians pear (Pyrus communis 'Williams'). Brazilian Journal of Development, 6(7), 51931-51940. 10.34117/bjdv6n7-732

Sozzi, G., \& Ramos, D. S. (2015). Avaliação do ágio no preço da energia convencional no mercado brasileiro de contratos de curto prazo: metodologia e aplicação. Revista Espaço Ciência, 22, 24.

Swami, S. B., \& Kalse, S. B. (2018). Jackfruit (Artocarpus heterophyllus): Biodiversity, nutritional contents and health. Bioactive Molecules in Food. Reference Series in Phytochemistry. Springer, Cham. English. 10.1007/978-3-319-54528-8_87-1

StatSoft, Inc. (2007). STATISTICA (data analysis software system), version 8.0. www.statsoft.com.

Tadini, C. C., Telis, V. R. N., Meirelles, A. J. A., \& Pessoa-Filho, P. A. (2016). Operações unitárias na indústria de alimentos.: Editora LTC, 652 p.

Wang, C. Y., \& Singh, R. P. (1978). Use of variable equilibrium moisture content in modeling rice drying. Transactions of the of the American Society of Agricultural, 11(78), 3001 .

Yagcioglu, A., Degirmencioglu, A., \& Cagatay, F. (1999). Drying characteristics of laurel leaves under different conditions. In: International Congress on Agricultural Mechanization and Energy, 7, Adana. Proceedings... Adana: Faculty of Agriculture, Cukurova University, 1999, p.565-569. 